\title{
HABILIDADES SOCIAIS E VARIÁVEIS SOCIODEMOGRÁFICAS EM ESTUDANTES DO ENSINO FUNDAMENTAL
}

\author{
Marina Bandeira \\ Sandra Silva Rocha\# \\ Lucas Cordeiro Freitas \\ Zilda Aparecida Pereira Del Prette ${ }^{\infty}$ \\ Almir Del Prette
}

\begin{abstract}
RESUMO. As habilidades sociais são consideradas situacionais, mas há poucos estudos no Brasil relacionando-as a variáveis sociodemográficas, especialmente com crianças. Investigou-se a importância e freqüência das habilidades sociais em relação a variáveis sociodemográficas, com 257 estudantes, de $1^{\mathrm{a}}$ a $4^{\mathrm{a}}$ série do ensino fundamental. Para avaliar habilidades sociais, utilizou-se o Sistema de Avaliação de Habilidades Sociais (SSRS) e, para o nível socioeconômico, o Critério Brasil. Participaram, como informantes, 185 pais e 12 professoras. Os resultados mostraram um efeito significativo do sexo, idade e indicadores socioeconômicos. Os melhores escores foram das meninas nas auto-avaliações e avaliação das professoras. A idade se correlacionou negativamente com auto-avaliação das habilidades sociais. Quanto maior a escolaridade dos pais e seu nível socioeconômico, maior o escore das habilidades sociais; quanto menor a importância atribuída pelos pais às habilidades sociais, menores os escores das crianças. O nível de habilidades sociais das crianças variou, portanto, em função de características sociodemográficas e sociais.
\end{abstract}

Palavras-chave: habilidades sociais, variáveis sociodemográficas, estudantes do ensino fundamental.

\section{SOCIAL SKILLS AND SOCIODEMOGRAPHIC VARIABLES IN ELEMENTARY SCHOOL STUDENTS}

\begin{abstract}
Social skills are situational, but a few researches evaluated its relation to sociodemographic variables in Brazil. This research investigated the social skills and its relation to sociodemographic variables in a sample of 257 elementary school students from $1^{\text {st }}$ to $4^{\text {th }}$ grades, in Brazil, using the SSRS scale (Social Skills Rating System). Socioeconomic levels were evaluated by "Criterio Brasil" scale. Participated in this research 185 parents e 12 teachers rating the children's social skills. Results showed significant differences in gender, the girls having higher scores in social skills than the boys. Social skills were significantly and positively correlated to social economic level and parents' level of education. Also, children from private schools had higher scores in social skills than those from public schools. Social skills were more frequent when considered important by parents. Age was negatively correlated to social skills, only in the self-evaluation scores. These results showed that the level of social skills in elementary school students vary according to sociodemographic and social variables.
\end{abstract}

Key words: Social skills, sociodemographic variables, elementary school students.

\section{HABILIDADES SOCIALES Y VARIABLES SOCIODEMOGRÁFICAS EN ESTUDIANTES DE ENSEÑANZA FUNDAMENTAL}

RESUMEN Las habilidades sociales son consideradas situacionales, pero hay pocos estudios en Brasil relacionándolas a variables sociodemográficas, especialmente con niños. Se investigó la importancia y frecuencia de las habilidades sociales en relación con variables sociodemográficas, con 257 estudiantes, de $1^{\circ}$ a $4^{\circ}$ año de enseñanza fundamental. Para evaluar

* Ph.D. Professora Adjunta da Universidade Federal de São João del Rei. Pesquisadora-bolsista do CNPq. Pós-doutorado pela McGill University e Université de Montréal.

\# Professora Assistente da Universidade Federal de São João del Rei. Mestre pela Universidade Federal do Rio de Janeiro.

II Aluno do curso de graduação de Psicologia da Universidade Federal de São João del Rei. Bolsista de Iniciação Científica do CNPq.

x Professora Titular da Universidade Federal de São Carlos. Pesquisadora-bolsista do CNPq. Pós-doutorado pela Universidade da Califórnia.

Æ Pesquisador-bolsista do CNPq. Doutor pela Universidade de São Paulo. 
habilidades sociales, se utilizó el Sistema de Evaluación de Habilidades Sociales (SSRS) y, para el nivel socioeconómico, el "Criterio Brasil". Participaron como informantes, 185 padres y 12 profesoras. Los resultados mostraron un efecto significativo de sexo, edad e indicadores socioeconómicos. Los mejores escores fueron de las chicas en las autoevaluaciones y evaluación de las profesoras. La edad se correlacionó negativamente con autoevaluación de las habilidades sociales. Cuanto mayor la escolaridad de los padres y su nivel socioeconómico, mayor el escore de las habilidades sociales; cuanto menor la importancia atribuida por los padres a las habilidades sociales, menores los escores de los niños. El nivel de habilidades sociales de los niños ha variado, por lo tanto, en función de características sociodemográficas y sociales.

Palabras-clave: habilidades sociales, variables sociodemográficas, estudiantes de enseñanza fundamental.

As habilidades sociais podem ser definidas como o conjunto dos desempenhos apresentados pelo indivíduo diante das demandas de uma situação interpessoal. Essas habilidades são aprendidas e as demandas para o seu desempenho variam em função do estágio de desenvolvimento do indivíduo e de variáveis situacional-culturais (Del Prette \& Del Prette, 1999).

Segundo Segrin e Flora (2000), a capacidade de um indivíduo para se comunicar e interagir com os outros de maneira efetiva e apropriada envolve um conjunto de habilidades complexas, tais como saber fazer perguntas e lidar com críticas, seguir regras, solicitar mudanças de comportamento e resolver situações interpessoais conflituosas. No contexto escolar, as habilidades sociais mais enfatizadas e valorizadas nos estudos internacionais dos últimos anos (Caldarella \& Merrel, 1997) podem ser agrupadas em cinco conjuntos de comportamentos: 1. relação com os companheiros (cumprimentar, elogiar, oferecer ajuda ou assistência, convidar para jogo de interação); 2. autocontrole (controlar o humor, seguir regras, respeitar limites); 3. habilidades sociais acadêmicas (envolver-se na tarefa, realizá-la de forma independente, seguir instruções); 4. ajustamento (seguir regras e comportar-se de acordo com o esperado); e 5. asserção (iniciar conversação, aceitar elogios, fazer convites).

A infância tem sido apontada como um período crítico para o desenvolvimento de habilidades sociais. Bussab (1999) defende que se a criança for apropriadamente estimulada nesse período, ela terá maiores probabilidades futuras de desenvolver interações sociais mais adequadas e reforçadoras. Embora o desenvolvimento da comunicação interpessoal do indivíduo tenha início desde o nascimento, esta se torna mais elaborada nas diversas etapas ao longo da sua vida (Del Prette \& Del Prette, 1999), sendo considerado fundamental em mudanças de uma situação para a outra, como, por exemplo, da permanência no lar para a escola, mesmo que em tempo parcial (Del Prette \& Del Prette, 2005).
A passagem do contexto familiar para o escolar exige da criança uma adaptação a novas demandas sociais, novas regras, interlocutores e papéis (Del Prette \& Del Prette, 1999). Espera-se que a criança, quando inserida neste novo contexto, amplie o seu repertório de habilidades sociais, que se torna progressivamente mais complexo. Além de contribuir para uma melhor adaptação da criança ao meio escolar, o aprimoramento de suas habilidades sociais pode prevenir o aparecimento de comportamentos agressivos (Baraldi \& Silvares, 2003) e de dificuldades de aprendizagem (Ferreira \& Marturano, 2002; Marinho, 2003). Os déficits de habilidades sociais estão associados a problemas psicossociais tais como depressão, ansiedade social, estresse e solidão (Segrin \& Flora, 2000). É importante investigar o repertório de habilidades sociais de crianças, visando à prevenção destes problemas.

As habilidades interpessoais possuem especi-ficidade situacional-cultural (Del Prette \& Del Prette, 1999), o que significa que os indivíduos podem ser socialmente habilidosos em um contexto e não em outro e, além disso, um mesmo desempenho social pode ser considerado competente em um contexto e não em outro. A maior parte dessas diferenças é determinada pela cultura, que define os padrões esperados para os diferentes contextos. Embora existam normas gerais para os desempenhos interpessoais, cada grupo ou classe social desenvolve normas particulares (Del Prette \& Del Prette, 1999; 2003a). Grande parte das normas que regulam a forma como as pessoas se comportam socialmente é estabelecida pelo grupo social a que pertencem, em termos de gênero, papéis sociais ou ocupacionais, filiação institucional (religião, seita, entidade filosófica ou política), contexto geográfico, etc. (Del Prette \& Del Prette, 1999; 2003a).

Há evidências, na literatura, de que a aquisição e o desenvolvimento das habilidades sociais estão associados a variáveis como sexo, idade e nível socioeconômico. Souza e Rodrigues (2002) encontraram diferenças significativas entre meninos e meninas de oito e nove anos, em um estudo de 
observação do comportamento, em situação de brincadeira, com videogravações. Os meninos apresentaram porcentagens mais altas das habilidades de abraçar o outro e iniciar contato do que as meninas, e estas, porcentagens mais altas na habilidade de pedir para entrar nas brincadeiras. Cecconello e Koller (2000) encontraram igualmente diferença entre os sexos, na avaliação da competência social e, em particular, da empatia, através do Teste de Histórias Incompletas (Mondell \& Tyler, 1981) e da Escala de Empatia (Bryant, 1982). O estudo foi realizado com 100 crianças pobres, com idades entre 6 e 9 anos, estudantes de $1^{\text {a }}$ a $3^{\text {a }}$ séries de escolas públicas da cidade de Porto Alegre. As meninas apresentaram um escore significativamente superior ao dos meninos na medida global de competência social, assim como na habilidade específica de empatia. Esse desempenho social superior das meninas, obtido nos dois estudos nacionais citados acima, foi igualmente encontrado por Gresham e Elliott (1990), em estudo com 2400 crianças estadunidenses, do Ensino Fundamental, utilizando a escala Social Skills Rating System (SSRS). As meninas apresentaram um escore de habilidades sociais significativamente superior ao dos meninos, nas avaliações feitas pelos professores, pelos pais e nas auto-avaliações das crianças.

Contrariamente aos resultados descritos acima, no estudo de Del Prette e Del Prette (2002) não foram observadas diferenças significativas em função do sexo, possivelmente devido ao instrumento de medida utilizado, que diferia daqueles adotados nos demais estudos citados. Foi utilizado o Inventário Multimídia de Habilidades Sociais em uma amostra de 406 alunos, de 7 a 13 anos de idade (com maior concentração de 8 a 10), cursando a $3^{\mathrm{a}}$ série do Ensino Fundamental de 6 escolas da rede estadual de ensino. $\mathrm{O}$ instrumento de medida utilizado é composto por 21 situações filmadas de interações sociais, cada uma delas com três alternativas de reação apresentadas pela personagem principal. Os autores sugeriram a realização de novos estudos, inclusive longitudinais, para verificar diferenças de sexo em habilidades sociais, em diferentes momentos do desenvolvimento infantil (Del Prette \& Del Prette, 2002).

Com relação ao nível socioeconômico, Lordelo (2002), investigando especificamente a freqüência de ocorrência de comportamentos verbais e não-verbais, em crianças de nível socioeconômico baixo e médio, encontrou diferenças significativas entre os grupos, em um estudo de observação de 148 crianças (81 de nível baixo e 67 de nível médio), filmadas em situação de brinquedo livre. As crianças de nível socioeconômico baixo apresentaram um número maior de interações sociais não-verbais, enquanto que as de nível médio emitiam maior número de interações verbais. Embora não se tenham avaliado categorias de habilidades sociais, constatou-se uma diferença no comportamento das crianças, em situações sociais, em função do nível socioeconômico. Esta diferença pode ser explicada, possivelmente, por um repertório verbal mais elaborado nas crianças de nível socioeconômico médio, corroborando a influência do ambiente na interação social.

Cecconello e Koller (2000) não encontraram diferenças significativas entre grupos de crianças de nível socioeconômico baixo e médio, com relação ao grau de competência social, avaliado através do Teste de Histórias Incompletas (Mondell \& Tyler, 1981). Este resultado negativo contrasta com os dados obtidos no estudo de Lordelo (2002), citado acima, talvez em função das diferenças nos instrumentos de medida utilizados nestas duas pesquisas. Dados de observação do comportamento podem ser mais sensíveis para detectar diferenças entre grupos.

No que se refere à influência da idade no nível de desenvolvimento das habilidades interpessoais, Cecconello e Koller (2000) encontraram diferenças significativas de competência social e de iniciativa em crianças de diversas faixas etárias, por meio do Teste de Histórias Incompletas (Mondell \& Tyler, 1981). As mais velhas (de 8 e 9 anos) apresentaram um nível mais elevado de iniciativa e competência social do que as mais novas (de 6 e 7 anos), indicando que com o aumento da idade as crianças tendem a se tornar mais socialmente competentes. Bryant (1982) também encontrou diferenças significativas em função da idade, ao investigar especificamente a empatia entre crianças e adolescentes de primeira, quarta e sétima séries, através de uma escala de empatia elaborada pelo próprio pesquisador. As crianças e os adolescentes da sétima série demonstraram ser mais empáticas do que as crianças da primeira e quarta séries. Esses resultados estão de acordo com muitas outras pesquisas, que mostram que a criança vai ampliando sua experiência de convivência ao participar de novos contextos que impõem demandas diferenciadas de desempenho, possibilitando-lhe assim novas aprendizagens (Garcia, 2001; Kliewer, 1991; Pavarino, 2004).

Considerando-se que a grande maioria das pesquisas, conforme a revisão da literatura nacional, não enfoca a relação entre habilidades sociais e variáveis demográficas e que, em alguns estudos, há divergência quanto ao sentido da relação encontrada (Bryant, 1982; Cecconello \& Koller, 2000; Del Prette \& Del Prette, 2002; Souza \& Rodrigues, 2002), é 
importante a continuidade de pesquisas nessa temática. Tendo em vista estas considerações, o objetivo da presente pesquisa foi verificar as relações entre as habilidades sociais e algumas características sociodemográficas: sexo, idade e nível socioeconômico.

\section{MÉTODO}

\section{Participantes}

Participaram desta pesquisa 257 crianças, de $1^{\mathrm{a}}$ a $4^{\mathrm{a}}$ séries de duas escolas públicas e uma particular de uma cidade do interior do Estado de Minas Gerais, totalizando 12 classes. Em cada escola, participou uma classe de cada série, indicada pela diretora, em função da disponibilidade das professoras em participar. A idade média das crianças foi de 8,62 anos $(D P .=1,49)$, sendo $111(43,2 \%)$ crianças do sexo feminino e 146 $(56,8 \%)$ do sexo masculino. Com relação ao nível socioeconômico, 4,9\% das crianças pertenciam à classe socioeconômica $\mathrm{A} 1,10,9 \%$ à classe $\mathrm{A} 2,12 \%$ à classe $\mathrm{B} 1,25 \%$ à classe $\mathrm{B} 2,33,2 \%$ à classe $\mathrm{C}, 13,6 \%$ à classe $\mathrm{D}$ e $0,5 \%$ à classe $\mathrm{E}$. As classificações do nível socioeconômico das crianças foram obtidas através do questionário Critério-Brasil.

Além disso, participaram também da pesquisa pais e professores, que avaliaram o repertório das crianças. Foram 185 pais, sendo $17(9,2 \%)$ do sexo masculino e $168(90,8 \%)$ do sexo feminino, com uma média de idade de 38,09 anos $(D P .=8,93)$. Os docentes eram todos do sexo feminino e tinham, em média, 43,16 anos de idade $(D P=5,06)$.

\section{Instrumentos de medida}

Foram utilizados dois instrumentos de medida: o Sistema de Avaliação de Habilidades Sociais (Social Skills Rating System ou SSRS - Gresham \& Elliott, 1990), traduzido e adaptado para o Brasil por Del Prette (2003b), e o questionário Critério Brasil para medir o nível socioeconômico.

Inventário SSRS: Este inventário avalia o repertório de habilidades sociais de crianças, por meio de três questionários, dirigidos aos pais, aos estudantes e aos professores. O questionário dos estudantes possui 35 itens que avaliam a freqüência das habilidades sociais. As alternativas de respostas estão dispostas em uma escala tipo Likert $(0=$ nunca, $1=$ algumas vezes e $2=$ muito freqüente).

O questionário para professores contém 30 itens que avaliam a frequiência e a importância das habilidades sociais das crianças, 18 itens que avaliam a freqüência de comportamentos problemáticos e 9 itens que avaliam a competência acadêmica dos estudantes. As alternativas de resposta estão dispostas em uma escala de freqüência, que varia de 0 a $2(0=$ nunca, $1=$ algumas vezes e $2=$ muito freqüente) e uma escala de importância, que também varia de 0 a $2(0=$ não importante, $1=$ importante e $2=$ muito importante). A escala de competência acadêmica possui 5 alternativas de respostas, que visam classificar os alunos com relação à sua turma, da seguinte maneira: $1=10 \%$ piores, $2=20 \%$ piores, $3=40 \%$ médios, $4=20 \%$ bons e $5=10 \%$ ótimos.

O questionário para pais possui 38 itens que avaliam a freqüência e a importância das habilidades sociais das crianças e 17 itens que avaliam a frequiência de comportamentos problemáticos. As pontuações das escalas de frequiência e de importância são idênticas às do questionário dos professores descrito acima.

Foi realizado o estudo da consistência interna das escalas de frequiência e de importância, com base no coeficiente alfa de Cronbach. Foram obtidos valores adequados de coeficiente alfa para as três escalas de frequiência (estudantes $=0,77$; pais $=0,86$; professores $=$ 0,92). Quanto à avaliação da importância, obteve-se um coeficiente alfa adequado apenas para a escala dos pais $($ alfa $=0,90)$. Por isto, serão analisados apenas os dados dos pais, para o grau de importância. O critério para retenção dos itens nas escalas foi um grau de correlação item total superior a 0,20.

Questionário Critério-Brasil: este questionário visa avaliar o nível socioeconômico. Possui 9 itens que avaliam o número de bens de consumo duráveis da família, um item que avalia o grau de instrução do chefe da família e um item que avalia o número de empregadas mensalistas na casa. Esta medida é estratificada em 5 classes, sendo elas: A (subdividida em A1 e A2), B (também subdividida em B1 e B2), C, $\mathrm{D}$ e E.

\section{Procedimento de coleta de dados}

Inicialmente, foram realizadas reuniões com as professoras e a diretora de cada escola para expor os objetivos do projeto e esclarecer as dúvidas. Em seguida, foram enviadas cartas de consentimento aos pais, solicitando-se a autorização para a participação dos seus filhos na pesquisa. Em reuniões, os três segmentos da amostra receberam todas as informações pertinentes à pesquisa e quanto ao uso dos dados, garantindo-se-lhes o anonimato e a liberdade de deixar de colaborar quando o quisessem.

A aplicação da escala nas crianças foi realizada em sala de aula. Os aplicadores estabeleciam, primeiramente, um "rapport" com as crianças, 
entregavam os questionários e faziam a leitura das instruções. Em seguida, as crianças verbalizavam o que haviam entendido e, em caso de dificuldade de compreensão, era concedida assistência individual. Os aplicadores liam cada uma das questões do questionário para as crianças para que elas respondessem. Ao final da aplicação, os aplicadores conferiam se todas as questões haviam sido respondidas uma única vez e se não havia nenhuma questão em branco. As crianças que não participavam da pesquisa foram dispensadas e acompanhadas pela professora em outras atividades, fora da sala de aula.

Os pais responderam ao questionário SSRS e ao Critério Brasil em reuniões agendadas, que ocorreram nas salas de aula das escolas. Após a leitura das instruções e esclarecimento das dúvidas, eles preenchiam os questionários em grupo e, em caso de dificuldades, recebiam assistência individual. Os pais, que não puderam comparecer às reuniões, foram novamente contatados para preenchimento do questionário, na escola ou em suas casas. $\mathrm{O}$ procedimento de aplicação da escala SSRS e do Critério Brasil para as professoras foi semelhante ao descrito para os pais, porém, elas responderam a uma escala SSRS para cada um de seus alunos.

\section{Análise dos dados}

Os dados foram digitados em um banco de dados no programa SPSS-PC (versão 7.5) para a realização das análises estatísticas. Para a comparação entre subgrupos de sujeitos, foi utilizado o Teste $t$ de Student para amostras independentes, assim como o teste ANOVA com teste post hoc Tukey. Para análise das relações entre variáveis, foi utilizado o coeficiente de correlação de Pearson.

\section{RESULTADOS}

Serão apresentados os resultados da freqüência de ocorrência das habilidades sociais e, em seguida, da importância atribuída pelos pais às habilidades sociais. Finalmente, serão relacionados os dados da frequiência e da importância das habilidades sociais.

\section{Avaliação da freqüência das Habilidades Sociais.}

\section{Habilidades sociais em função do gênero}

Os escores de habilidades sociais e seus desviospadrão, em função do gênero, se encontram na Tabela 1.

Tabela 1. Médias, Desvios-Padrão e Níveis de Significância das Habilidades Sociais em Função do Gênero e do Tipo de Escola, Obtidos Através da Avaliação dos Estudantes, Pais e Professoras

\begin{tabular}{llcccccc}
\hline \multirow{2}{*}{ Variáveis } & \multirow{2}{*}{ Grupos } & \multicolumn{2}{c}{ Estudantes } & \multicolumn{2}{c}{ Pais } & \multicolumn{2}{c}{ Professoras } \\
\cline { 3 - 8 } & & $\boldsymbol{N}$ & Média $(\boldsymbol{D P})$ & $\boldsymbol{N}$ & Média $(\boldsymbol{D P})$. & $\boldsymbol{N}$ & Média $(\boldsymbol{D P})$ \\
\hline \multirow{2}{*}{ Sexo } & Masculino & 146 & $47,29(7,48)$ & 101 & $47,26(9,05)$ & 146 & $39,87(9,97)$ \\
\multirow{2}{*}{ Tipo de Escola } & Feminino & 110 & $51,18^{*}(6,32)$ & 84 & $49,25(9,77)$ & 110 & $44,94^{*}(9,12)$ \\
& Pública & 181 & $47,90(7,25)$ & 143 & $47,01(9,42)$ & 181 & $39,46(9,60)$ \\
& Particular & 75 & $51,52^{*}(6,65)$ & 42 & $52,09 *(8,34)$ & 75 & $48,30^{*}(7,69)$ \\
\hline
\end{tabular}

${ }^{*} p<0,05$

Pode-se observar, na Tabela 1 , que tanto na auto-avaliação dos estudantes quanto na avaliação das professoras, as meninas apresentaram uma média significativamente maior de habilidades sociais do que os meninos $(t=-4,392$ e $-4,172$; $p<0,05)$; no entanto, na avaliação dos pais, não houve diferença significativa entre os sexos $(t=-$ $1,430 ; p>0,05)$.

\section{Habilidades sociais e nível socioeconômico}

O nível socioeconômico foi avaliado através de três indicadores. No que se refere ao questionário Critério Brasil, obteve-se uma correlação positiva significativa entre seu resultado e o escore global de habilidades sociais, avaliadas pelos pais $(r=0,28$; $p<0,05)$, professoras $(r=0,39 ; p<0,05)$ e crianças $(r=$ $0,22 ; \quad p<0,05)$. Quanto mais elevado o nível socioeconômico, maiores os escores de habilidades sociais.

A escolaridade dos pais obteve uma correlação positiva significativa com o escore de habilidades sociais, na avaliação das professoras ( $r=0,37$; $p<0,05)$, dos pais $(r=0,20 ; p<0,05)$ e das crianças $(r=$ $0,15 ; p<0,05)$. Quanto maior era o grau de escolaridade dos pais, maior era a média de habilidades sociais dos estudantes.

Quanto ao tipo de escola, os escores dos sujeitos se encontram na Tabela 1. As crianças da escola particular apresentaram uma média significativamente mais elevada de habilidades sociais do que as das escolas públicas, nas três avaliações efetuadas, (estudantes: $t=-3,71$; pais $=-3,14$; professoras $=-7,08$; $p<0,05)$. 


\section{Habilidades sociais e idade}

Considerando-se a auto-avaliação feita pelos estudantes, obteve-se uma correlação negativa significativa $(r=-0,20 ; p<0,05)$ entre a idade e o escore global de habilidades sociais. Quanto menor era a idade do estudante, maior era seu escore global de habilidades sociais. As correlações, porém, não foram significativas para os dados da avaliação dos pais e professoras ( $r=-0,06$ e $-0,09 ; p>0,05)$.

\section{Habilidades sociais por série}

A Tabela 2 apresenta as médias e os desviospadrão das habilidades sociais dos estudantes em cada série. A Tabela 3 apresenta os níveis de significância obtidos nas comparações entre as séries.

Tabela 2. Médias e Desvios-Padrão das Habilidades Sociais dos Estudantes em Cada Série, Obtidas Através da Avaliação dos Estudantes, Pais e Professoras

\begin{tabular}{|c|c|c|c|c|c|c|}
\hline \multirow{2}{*}{ Série } & \multicolumn{2}{|c|}{ Estudantes } & \multicolumn{2}{|c|}{ Pais } & \multicolumn{2}{|c|}{ Professoras } \\
\hline & $N$ & Média $(D P)$ & $N$ & Média $(D P)$ & $N$ & Média $(D P)$ \\
\hline $1^{a}$ Série & 53 & $50,11(8,13)$ & 36 & $45,80(8,27)$ & 53 & $41,53(9,73)$ \\
\hline $2^{a}$ Série & 70 & $51,48(5,89)$ & 47 & $50,49(9,73)$ & 69 & $40,13(8,22)$ \\
\hline $3^{\text {a }}$ Série & 60 & $46,08(7,35)$ & 41 & $47,78(9,81)$ & 61 & $41,74(9,68)$ \\
\hline $4^{a}$ Série & 73 & $48,08(6,80)$ & 61 & $48,03(9,35)$ & 73 & $44,52(11,33)$ \\
\hline TOTAL & 256 & $48,96(7,25)$ & 185 & $48,16(9,41)$ & 256 & $42,05(9,92)$ \\
\hline
\end{tabular}

A ANOVA mostrou que as quatro séries diferiram significativamente entre si $(F=7,27 ; p<0,05)$. O teste post hoc Tukey mostrou a direção desta diferença, indicando que, na auto-avaliação dos estudantes, os escores de habilidades sociais das crianças da $1^{\mathrm{a}}$ e da $2^{\mathrm{a}}$ séries foram superiores aos da $3^{\mathrm{a}}$ série $(p<0,05)$. As crianças da $2^{\mathrm{a}}$ série apresentaram uma média de habilidades sociais significativamente superior à das crianças da $4^{\mathrm{a}}$ série $(p<0,05)$.

Tabela 3. Níveis de Significância das Habilidades Sociais dos Estudantes em Cada Série, Obtidos Por Meio da Avaliação dos estudantes, Pais e Professoras.

\begin{tabular}{|c|c|c|c|c|}
\hline \multirow{2}{*}{ Série } & \multirow{2}{*}{ Série } & Estudantes & Pais & Professoras \\
\hline & & Nível de Significância $(p)$ & Nível de Significância $(p)$ & Nível de Significância $(p)$ \\
\hline \multirow{3}{*}{$1^{\text {a }}$ Série } & $2^{\mathrm{a}}$ Série & 0,70 & 0,10 & 0,86 \\
\hline & $3^{\text {a }}$ Série & $0,01^{*}$ & 0,79 & 0,99 \\
\hline & $4^{a}$ Série & 0,37 & 0,67 & 0,86 \\
\hline \multirow{2}{*}{$2^{a}$ Série } & $3^{\text {a }}$ Série & $0,00^{*}$ & 0,52 & 0,78 \\
\hline & $4^{a}$ Série & $0,01 *$ & 0,53 & $0,03 *$ \\
\hline $3^{\text {a }}$ Série & $4^{a}$ Série & 0,35 & 0,99 & 0,36 \\
\hline
\end{tabular}

$* p<0,05$

A ANOVA mostrou que, na avaliação das professoras, as quatro séries diferiram significativamente entre si $(F=2,47 ; p<0,05)$. O teste post hoc Tukey indicou que as crianças da $4^{\text {a }}$ série apresentaram escores superiores aos das crianças da $2^{\mathrm{a}}$ série $(p<0,05)$. No caso da avaliação dos pais, não houve diferença significativa $(F=1,75 ; p>0,05)$.

\section{Avaliação da importância das habilidades sociais}

Os dados referentes à importância atribuída pelos pais às habilidades sociais das crianças foram relacionados com as variáveis sociodemográficas, descritas abaixo:

\section{Importância das habilidades sociais e gênero}

Não foram observadas diferenças de gênero com relação aos dados sobre a importância ( $t=0,44$; $p>0,05)$. Os pais atribuem importância às habilidades sociais igualmente para as meninas e meninos.

\section{Importância das habilidades e nível socioeconô- mico}

A importância atribuída pelos pais às habilidades sociais foi analisada para os três indicadores de nível socioeconômico. Para os dados do questionário Critério Brasil, obteve-se uma correlação positiva significativa ( $r=0,20 ; p<0,05)$ com o grau de importância. Quanto mais elevado era o nível socioeconômico dos pais, maior a importância atribuída às habilidades sociais.

A escolaridade dos pais se correlacionou significativamente com os escores de importância ( $r=$ $0,18 p<0,05)$. Quanto maior o nível de escolaridade 
dos pais, maior o grau de importância atribuída por eles às habilidades sociais. Quanto ao tipo de escola, obteve-se uma diferença significativa, tendo os pais das crianças da escola particular atribuído maior importância às habilidades sociais do que os das escolas públicas $(t=-4,03 ; p<0,05)$.

\section{Importância das habilidades e idade}

A correlação entre a importância atribuída pelos pais às habilidades sociais e a idade das crianças não foi significativa $(r=-0,11 ; p>0,05)$; portanto, o grau de importância não variou em função da idade das crianças.

\section{Relações entre importância e freqüência das habilidades sociais}

Houve uma correlação positiva significativa entre os escores de importância e de freqüência das habilidades sociais. Quanto maior era a importância atribuída pelos pais a estas habilidades, maior a freqüência de sua emissão pelas suas crianças. Este resultado foi observado na avaliação de habilidades sociais feita pelos pais $(r=0,23 ; p<0,05)$ e pelos estudantes $(r=0,15 ; p<0,05)$, mas não quando feita pelas professoras $(r=0,14 ; p>0,05)$.

\section{DISCUSSÃO}

Os resultados obtidos na presente pesquisa confirmam os dados encontrados na literatura da área. Com relação ao sexo, os resultados mostraram que as meninas apresentaram uma maior freqüência de habilidades sociais do que os meninos, na autoavaliação e na avaliação das professoras. Esses resultados corroboram os dados de pesquisas nacionais e de outros países (Cecconello \& Koller, 2000; Gresham \& Elliott, 1990), que também utilizaram instrumentos semelhantes (tipo lápispapel) e se basearam em escores globais de competência social, como na presente pesquisa.

Estes resultados diferem dos de outras pesquisas (Del Prette \& Del Prette, 2002; Souza \& Rodrigues, 2002), que não encontraram diferenças entre os sexos, o que possivelmente se explique pela metodologia utilizada (inventário multimídia e observação direta, respectivamente, sendo que o segundo baseou-se em categorias isoladas das habilidades sociais). Conforme a literatura da área, dados obtidos por meio de diferentes instrumentos de medida podem não apresentar correlação entre os seus indicadores (Lipp, Haythornthwaite \&
Anderson, 1996). As avaliações dos pais não mostraram diferenças entre os dois sexos, o que evidencia o peso dos critérios adotados pelos diferentes informantes e a importância da avaliação multimodal nessa área (Del Prette \& Del Prette, 2002, 2003a).

Com relação ao nível socioeconômico, a presente pesquisa observou uma relação positiva significativa com o nível das habilidades sociais das crianças. Lordelo (2002) também encontrou diferenças no repertório de crianças, em função do seu nível socioeconômico, embora se referissem apenas à freqüência de comportamentos verbais e não-verbais em interações sociais, e não a uma avaliação de habilidades sociais. Entretanto, a pesquisa de Cecconello e Koller (2000), por sua vez, não encontrou diferenças significativas de competência social entre crianças de níveis socioeconômicos baixo e médio, o que pode ter sido provocado por limitações metodológicas já levantadas na introdução deste artigo.

No que se refere às relações entre idade e habilidades sociais, as crianças mais novas apresentaram maior grau de habilidades sociais, contrariando os dados de duas pesquisas, assim como as teorias sobre as habilidades sociais. Por exemplo, Cecconello e Koller (2000) encontraram resultados opostos: as crianças mais velhas (da faixa etária de 8 e 9 anos) apresentaram maior competência social e iniciativa do que as mais novas (da faixa etária de 6 e 7 anos). Isto talvez se explique pelo fato de os autores terem usado um tipo de medida muito diferente da adotada aqui (teste de histórias a serem completadas). Bryant (1982) também observou que as crianças da sétima série apresentavam um maior grau de empatia do que as da primeira e quarta séries, o que diverge da presente pesquisa, talvez pelo fato de o autor ter comparado crianças de faixa etária mais ampla, e não a faixa mais restrita e baixa usada na presente pesquisa (primeira a quarta séries). É possível ainda que os resultados encontrados aqui se expliquem pelo fato de a auto-avaliação das crianças refletir mais a sua autopercepção do que a presença das habilidades sociais no seu repertório.

Assim, os resultados encontrados na presente pesquisa, sobre as relações entre idade e habilidades sociais, não confirmam os dados da literatura da área, que enfatizam uma elaboração crescente desse repertório (Del Prette \& Del Prette, 1999; Kliewer, 1991;). Duas hipóteses podem ser 
levantadas para explicar esta divergência. A primeira evoca a diferença dos instrumentos de medida como possível explicação. A segunda aponta para uma possível especificidade própria desta amostra, enquanto cidade do interior, onde as crianças se movimentam mais precocemente de um ambiente para o outro. Todas essas variáveis teriam que ser melhor investigadas, para se obterem respostas conclusivas. De qualquer forma, tanto o resultado obtido neste estudo como essas hipóteses estão de acordo com o caráter situacional das habilidades interpessoais, destacando-se a influência dos contextos social e cultural na aquisição e desempenho das mesmas.

Quanto à comparação entre as séries, os resultados foram contraditórios. $\mathrm{Na}$ avaliação das professoras, as crianças da $4^{\mathrm{a}}$ série apresentaram mais habilidades sociais que as da $2^{\mathrm{a}}$ série, enquanto na auto-avaliação ocorreu o contrário. Esta contradição poderia ser explicada pelo fato de as professoras terem avaliado habilidades sociais mais estreitamente relacionadas ao desempenho acadêmico, o que não ocorreu nas auto-avaliações. Outra hipótese é a dos diferentes critérios de avaliação utilizados por diferentes informantes.

A importância atribuída pelos pais às habilidades sociais apresentou uma relação significativa com os indicadores socioeconômicos. Quanto mais altos o nível socioeconômico e a escolaridade dos pais, maior a importância atribuída por eles às habilidades sociais. Além disso, os pais das crianças da escola particular atribuíram maior importância às habilidades sociais do que os das escolas públicas. A importância atribuída pelos pais às habilidades sociais se relacionou com uma maior freqüência destas habilidades nas crianças.

\section{CONCLUSÕES}

Os resultados obtidos na presente pesquisa mostraram importantes diferenças entre grupos de crianças com relação às habilidades sociais, permitindo apontar as que necessitam, preferencialmente, de intervenções que visem ao treinamento destas habilidades. Os resultados destacam ainda as variáveis que estão associadas ao desenvolvimento das habilidades sociais.

Pode-se concluir que as habilidades sociais das crianças variaram significativamente em função de características sociodemográficas e que estas relações confirmaram, em geral, os dados da literatura da área, com alguns resultados discrepantes, explicáveis a partir da especificidade situacional das habilidades sociais. Com relação ao gênero, observou-se uma maior necessidade de os meninos serem acompanhados em sua aquisição de habilidades sociais, visando à prevenção de problemas de comportamento e a um melhor desenvolvimento adaptativo a médio e longo prazo, já que a competência social constitui um fator de proteção importante (Del Prette \& Del Prette, 2005).

Observou-se ainda a necessidade de uma maior atenção ao repertório de habilidades sociais de crianças de nível socioeconômico baixo, de escola pública e daquelas cujos pais possuem baixa escolaridade. $\mathrm{O}$ fato de esses pais valorizarem menos as habilidades sociais pode estar relacionado à situação de risco das famílias, que vivem em contato mais direto com a violência e com menos acesso às informações (presentes em revistas e jornais) sobre a importância dessas habilidades nas sociedades atuais.

A aquisição destas habilidades, no período escolar, pode contribuir para evitar o aparecimento de comportamentos problemáticos (Baraldi \& Silvares, 2003) e de dificuldades de aprendizagem (Del Prette \& Del Prette, 2003a). Portanto, trata-se de um repertório importante para o ajustamento acadêmico e social dos alunos, podendo ter repercussões em sua vida posterior. Além de novas questões de pesquisa, os resultados deste estudo apontam para várias implicações práticas, em termos da prevenção de problemas e da promoção do desenvolvimento infantil.

\section{REFERÊNCIAS}

Baraldi, D. M. \& Silvares, E. F. M. (2003). Treino de habilidades sociais em grupo com crianças agressivas, associado à orientação dos pais: análise empírica de uma proposta de atendimento. Em A. Del Prette \& Z. A. P. Del Prette (Org.), Habilidades sociais, desenvolvimento $e$ aprendizagem: questões conceituais, avaliação $e$ intervenção (pp. 235-258). Campinas: Alínea.

Bryant, B. K. (1982). An index of empathy for children and adolescents. Child Development, 53, 413- 425.

Bussab, V. S. (1999). Da criança ao adulto: que faz do ser humano o que ele é? Em A. M. Carvalho (Org.), O mundo social da criança: natureza e cultural em ação. São Paulo: Casa do Psicólogo.

Caldarella, P. \& Merrel, R. (1997). Common dimensions of social skills of children and adolescents: A taxonomy of 
positive behaviors. School Psychology Review, 26, 264278.

Cecconello, A. M. \& Koller, S. H. (2000). Competência social e empatia: um estudo sobre resiliência com crianças em situação de pobreza. Estudos de Psicologia, 5(1), 71-93

Del Prette, Z. A. P. \& Del Prette, A. (1999). Psicologia das habilidades sociais: terapia e educação. Petrópolis: Vozes.

Del Prette, Z. A P. \& Del Prette, A. (2002). Avaliação de habilidades sociais de crianças com um inventário multimídia: indicadores sociométricos associados a frequiência versus dificuldade. Psicologia em Estudo, 7(1), 61-73.

Del Prette, Z. A. P. \& Del Prette, A. (2003a). Habilidades sociais e dificuldade de aprendizagem: teoria e pesquisa sob um enfoque multimodal. Em A Del Prette \& Z. A. P. Del Prette (Orgs.), Habilidades sociais, desenvolvimento e aprendizagem: questões conceituais, avaliação $e$ intervenção (pp 167-206). Campinas: Alínea.

Del Prette, Z. A. P. \& Del Prette, A. (2003b). Prevenção de dificuldades de aprendizagem $e$ da violência: desenvolvendo tecnologia para avaliação e promoção de habilidades. (Relatório de auxílio à pesquisa no exterior pós-doutorado). São Paulo: FAPESP.

Del Prette, Z. A. P. \& Del Prette, A. (2005). Psicologia das habilidades sociais na infância: teoria e prática. Petrópolis: Vozes.

Ferreira, M., \& Marturano, E. (2002). Ambiente familiar e os problemas de comportamento apresentados por crianças com baixo desempenho escolar. Psicologia Reflexão $e$ Crítica, 15(1), 1-11.

Garcia, F. A. (2001). Investigando diferentes indicadores de empatia em meninos e sua relação com a empatia e ações educativas dos pais. Dissertação de Mestrado NãoPublicada, Programa de Pós-Graduação em Psicologia, Universidade de São Paulo, Ribeirão Preto.
Gresham, F. M. \& Elliott, S. N. (1990). Social Skills Rating System: Manual. Circle Pines: American Guidance Service.

Kliewer, W. (1991). Coping in middle childhood: Relations to competence, type A behavior, monitoring, blunting and locus of control. Developmental Psychology, 27, 689-697.

Lipp, M. N., Haythornthwaite, J. \& Anderson, D. E. (1996). Medidas diversas da assertividade em adultos. Estudos de Psicologia, 13(1), 19-26.

Lordelo, E. R. (2002). Interação social e responsividade em ambientes doméstico e de creche: cultura e desenvolvimento. Estudos de Psicologia, 7(2), 343-350.

Marinho, M. (2003). Comportamento anti-social infantil: questões teóricas e de pesquisa. Em Z. A. P. Del Prette \& A. Del Prette (Orgs.), Habilidades sociais, desenvolvimento e aprendizagem (pp. 61-81). Campinas: Alínea.

Mondell, S. \& Tyler, F. B. (1981). Child psychosocial competence and its measurement. Journal of Pediatric Psychology, 6, 145-154.

Pavarino, M. G. (2004). Agressividade e empatia: um estudo com crianças pré-escolares. Dissertação de Mestrado NãoPublicada, Programa de Pós-Graduação em Educação Especial, Universidade Federal de São Carlos, São Carlos.

Souza, F. \& Rodrigues, M. M. P. (2002). A segregação sexual na interação de crianças de 8 e 9 anos. Psicologia: reflexão e crítica, 15(3), 489-496.

Segrin, C. \& Flora, J. (2000). Poor social skills are a vulnerability factor in the development of psychosocial problems. Human Communication Research, 26(3), 489514.

Recebido em 17/04/2005 Aceito em 19/09/2005

Endereço para correspondência: Marina Bandeira. Departamento de Psicologia - UFSJ, Praça Dom Helvécio, 74, Bairro Fábricas, CEP: 336.301-160, São João del Rei - MG. E-mail: bandeira@ufsj.edu.br 